Supporting Information

Tungsten Nitride/Carbon Cloth as Bifunctional Electrode for Effective

\title{
Polysulfide Recycling
}

Yange Wang, ${ }^{\mathrm{a}, \mathrm{b}}$ Rongjie Luo, ${ }^{\mathrm{a}, \mathrm{b}}$ Yingge Zhang, ${ }^{\mathrm{a}, \mathrm{b}}$ Yan Guo, ${ }^{\mathrm{a}, \mathrm{b}}$ Yang Lu, ${ }^{\mathrm{a}, \mathrm{b}}$ Xianming

Liu, ${ }^{\mathrm{c}}$ Jang-Kyo Kim ${ }^{\mathrm{d}}$ and Yongsong Luo ${ }^{\mathrm{a}}$, b, *

a School of Physics and Electronic Engineering, Xinyang Normal University, Xinyang 464000, P. R. China.

b Key Laboratory of Microelectronics and Energy of Henan Province, Xinyang Normal University, Xinyang

464000, P. R. China.

${ }^{\mathrm{c}}$ College of Chemistry and Chemical Engineering, Luoyang Normal University, Luoyang 471934, P. R. China.

${ }^{d}$ Department of Mechanical and Aerospace Engineering, Hong Kong University of Science and Technology, Clear Water Bay, Kowloon, Hong Kong, P. R. China.

\footnotetext{
* To whom correspondence should be addressed: Tel./fax: +86 0376 6390801, E-mail: ysluo@xynu.edu.cn (Y. S. Luo), ORCID: 0000-0002-8000-3126.
} 


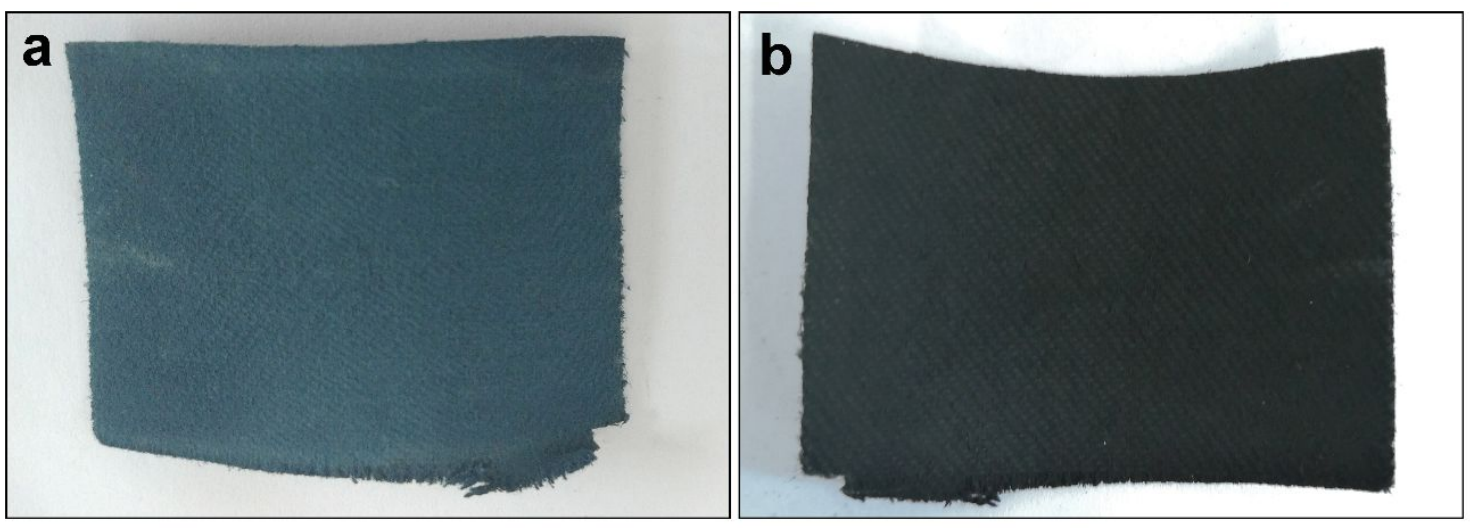

Figure S1. Photographs of $\mathrm{WO}_{3} / \mathrm{CC}$ (a) and WN/CC (b). 


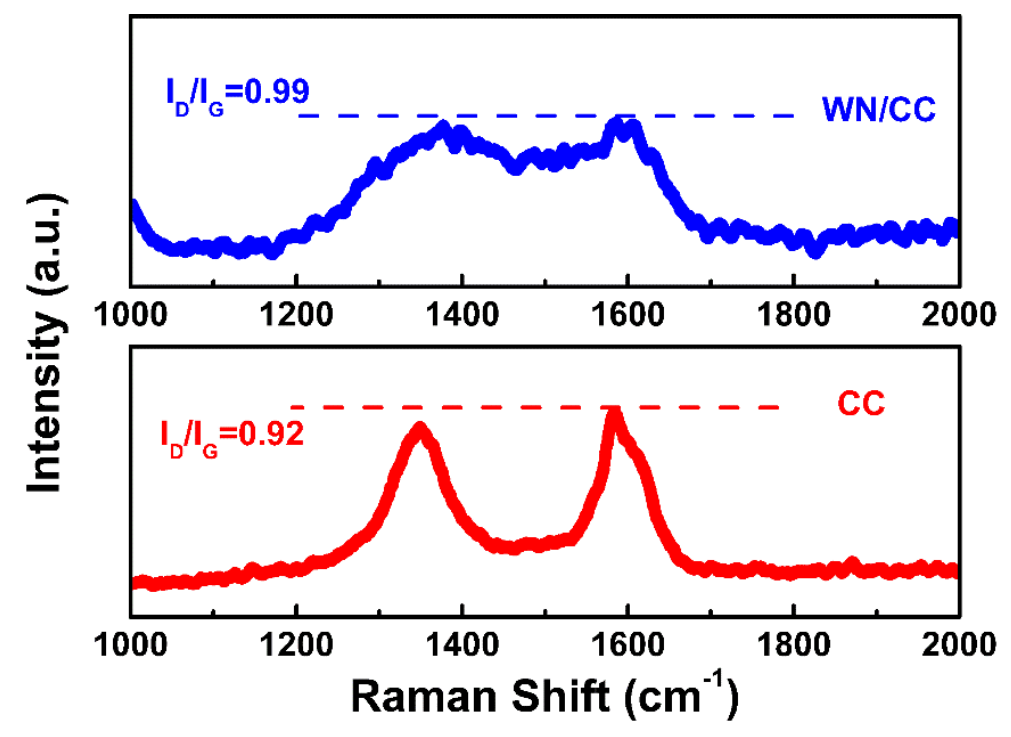

Figure S2. Raman spectra of WN/CC and CC. 


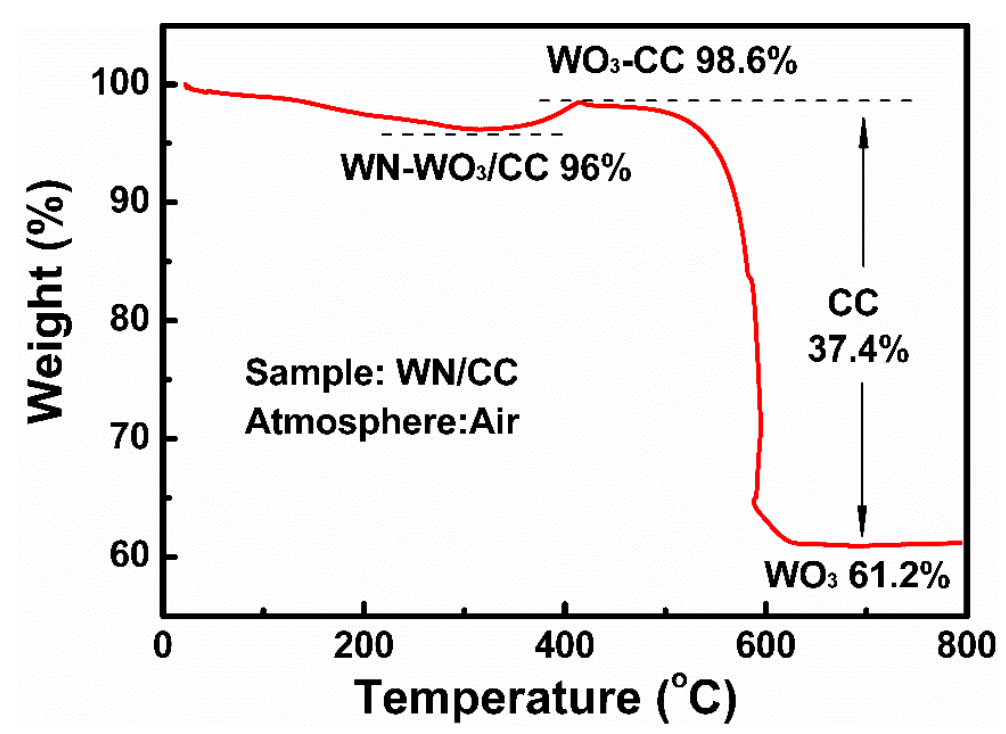

Figure S3. TG curve of the WN/CC composites. 

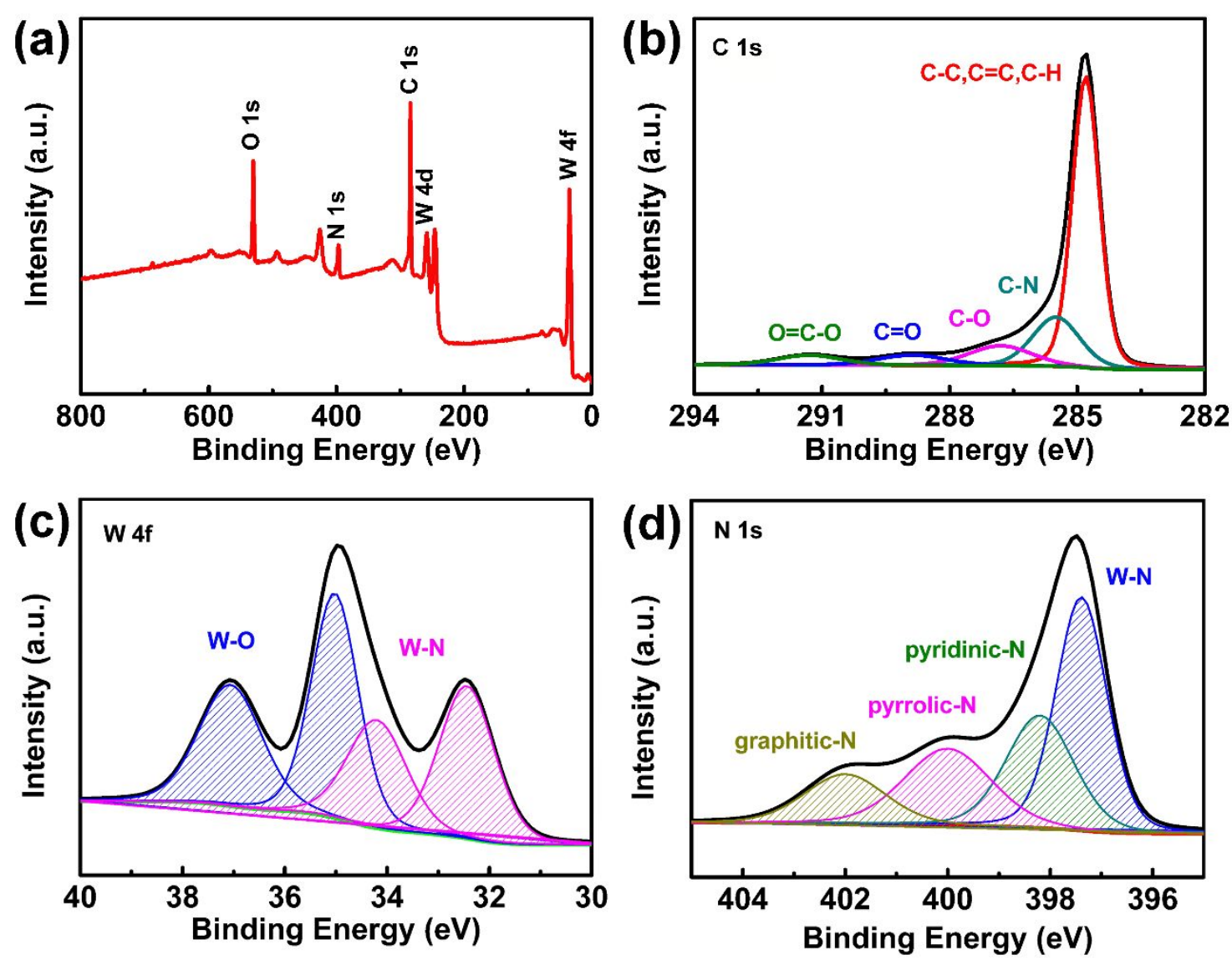

Figure S4. XPS survey spectrum of WN/CC (a). Deconvoluted C 1s (b), W 4f (c) and N 1s (d) spectra. 


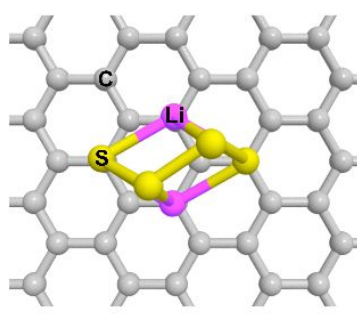

$0.62 \mathrm{eV}$

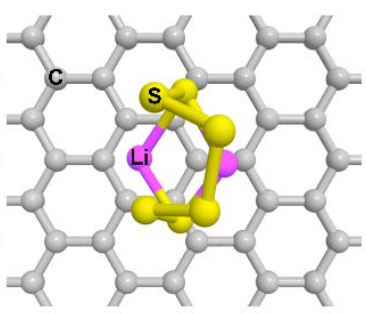

$0.71 \mathrm{eV}$

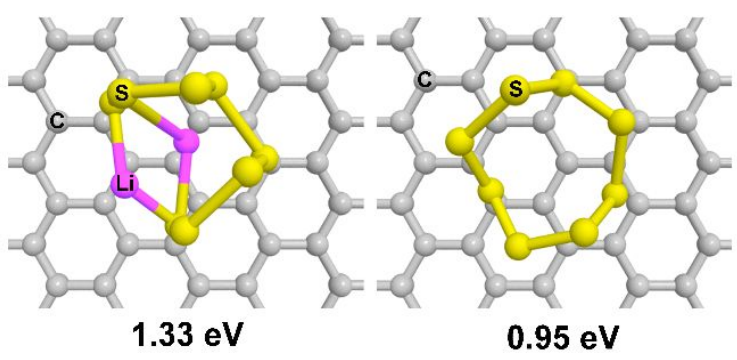

$1.33 \mathrm{eV}$

Figure S5. The optimized geometrical structures of $\mathrm{Li}_{2} \mathrm{~S}_{\mathrm{x}}(\mathrm{x}=4,6,8)$ and $\mathrm{S}_{8}$ adsorbed on carbon cloth. 


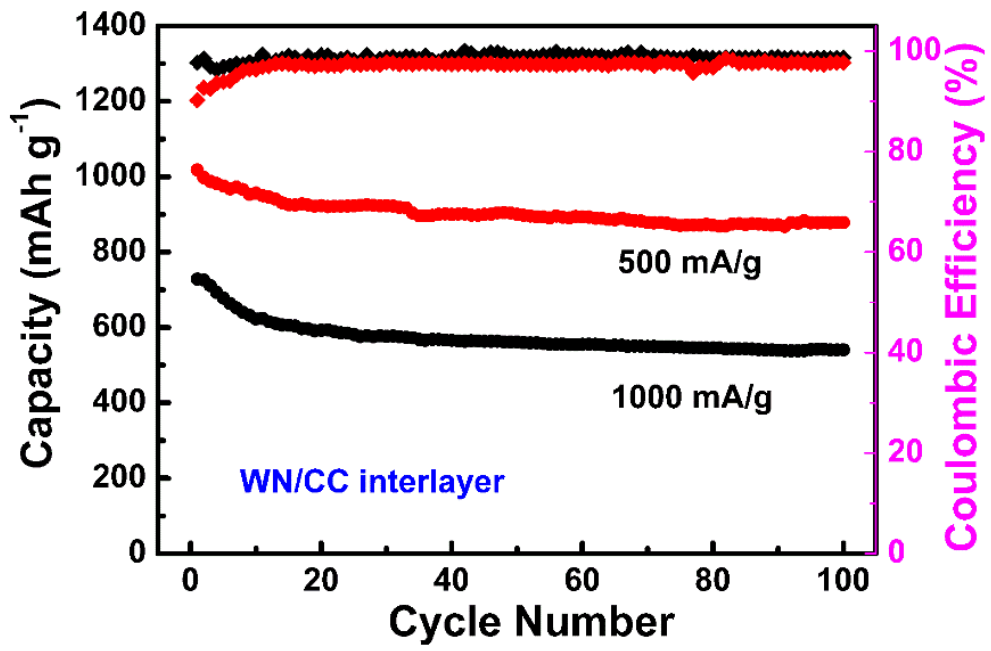

Figure S6. The cycle performances of the cell with WN/CC interlayer at $500 \mathrm{~mA} \mathrm{~g}^{-1}$ and $1000 \mathrm{~mA} \mathrm{~g}^{-1}$. 

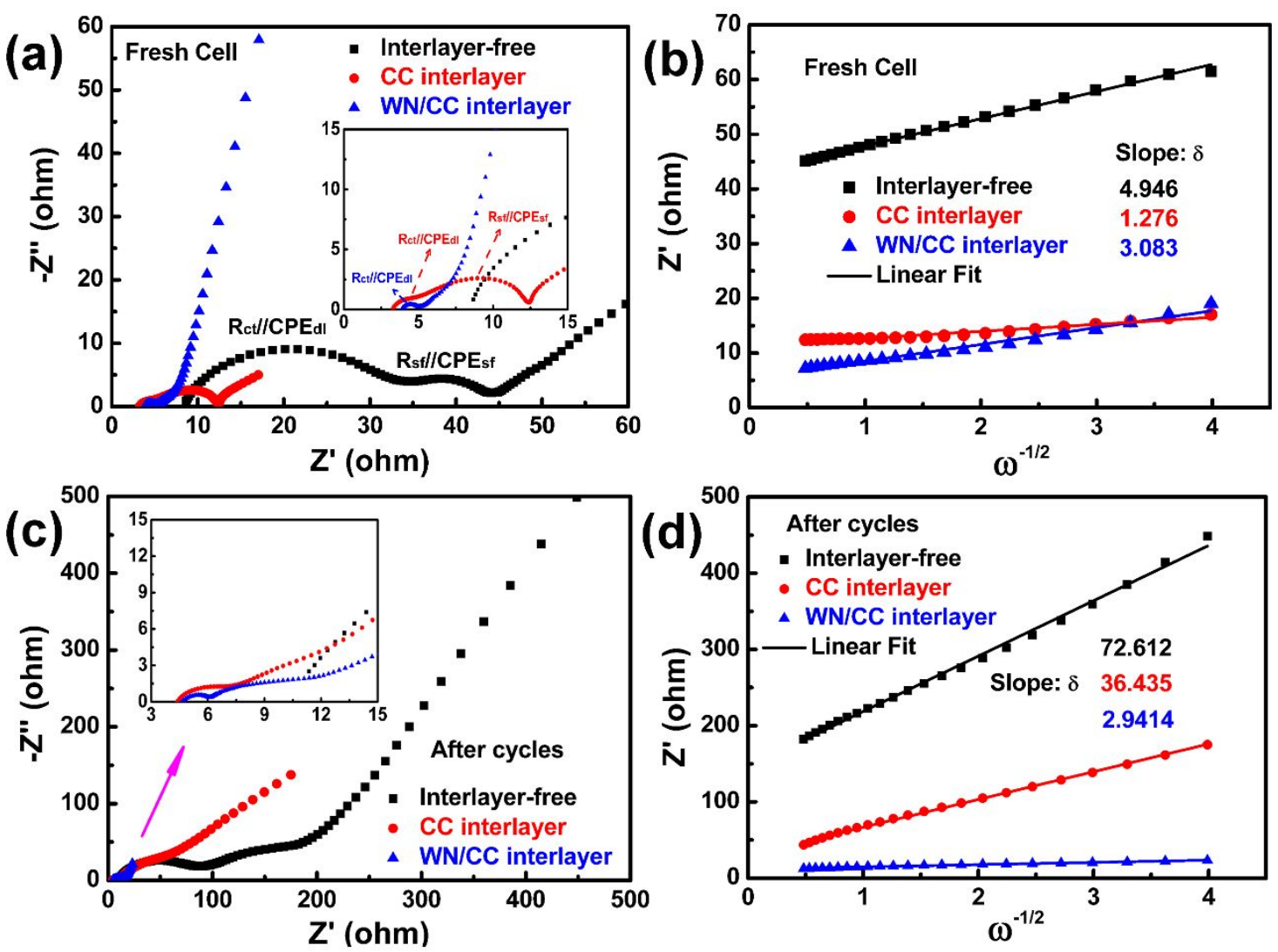

(e)
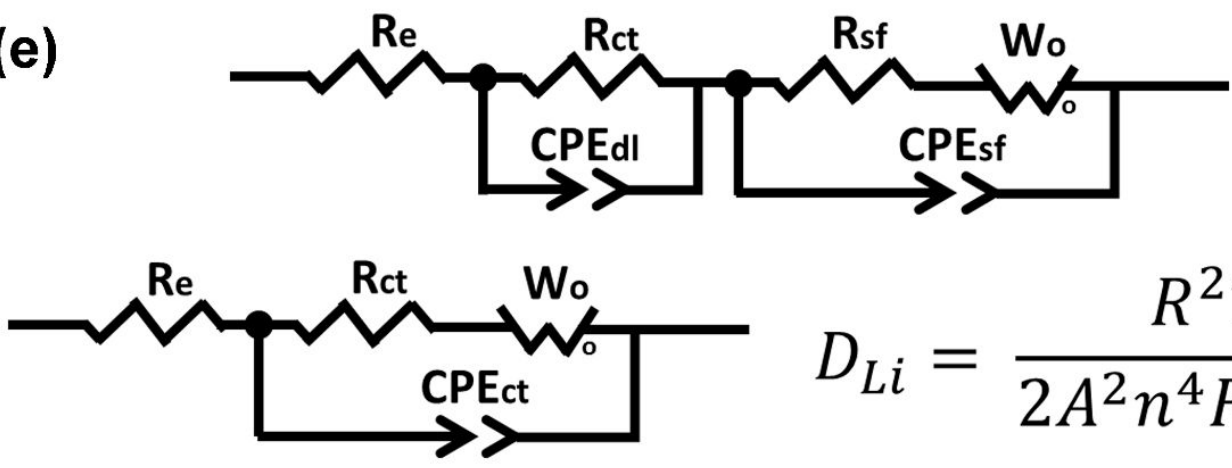

$$
D_{L i}=\frac{R^{2} T^{2}}{2 A^{2} n^{4} F^{4} C^{2} \delta^{2}}
$$

Figure S7. Nyquist plots (a and c) and Warburg plots of (b and d) of the cells with different interlayers before ( $a$ and $b$ ) and after 500 cycles (c and d). (e) The equivalent circuits of the cells with different interlayers. The inset of (a) and (c) are partial magnification of the Nyquist plots. 


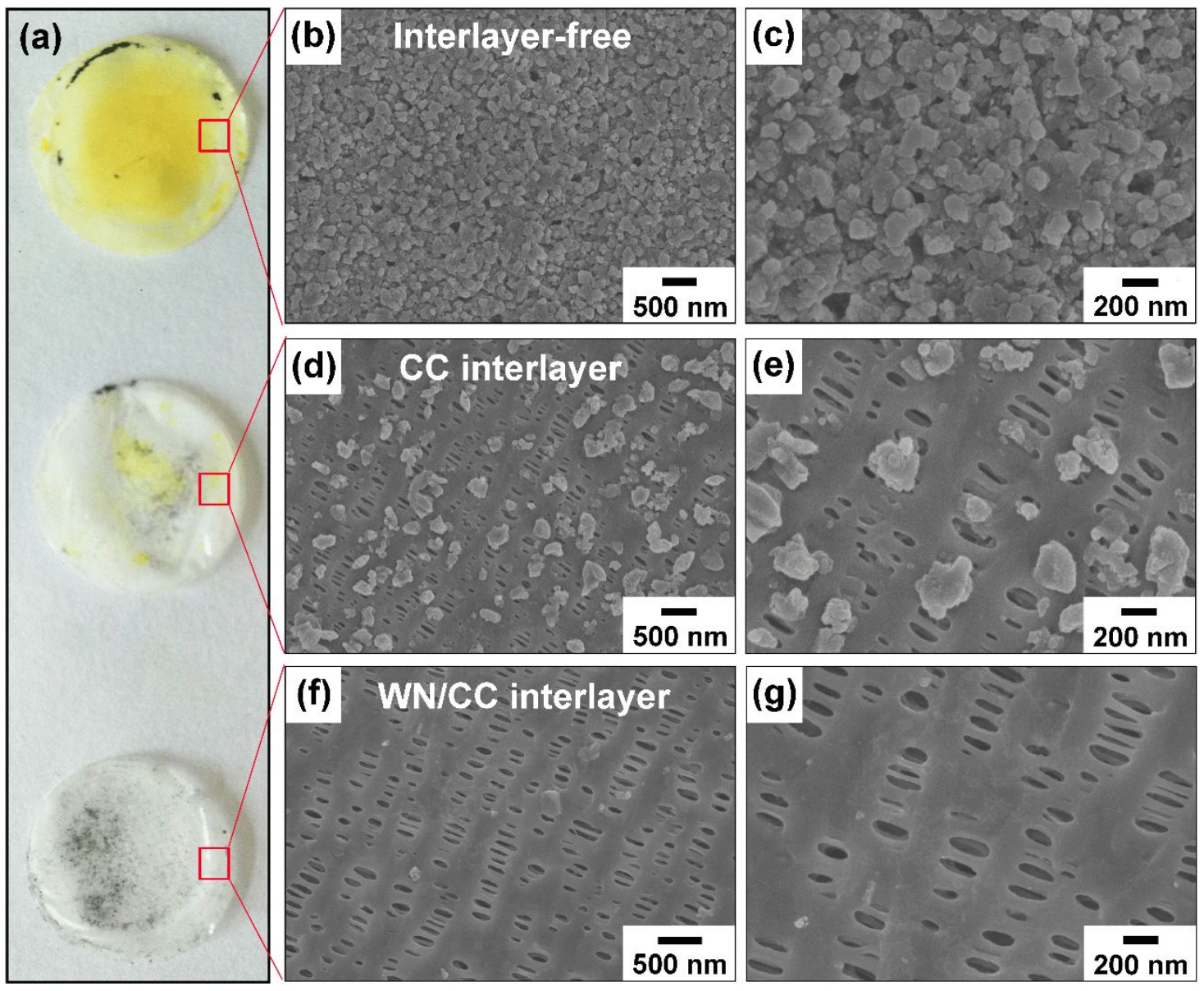

Figure S8. Digital images (a) and SEM images of the separator surfaces in cells without interlayer (b and c), with a CC interlayer ( $\mathrm{d}$ and e) and a WN/CC composite interlayer ( $\mathrm{f}$ and $\mathrm{g}$ ). 
Table S1. Comparison of electrochemical performance of LSBs with different types of separators/interlayers.

\begin{tabular}{|c|c|c|c|c|c|}
\hline Interlayer & $\begin{array}{c}\text { Cycle } \\
\text { number }\end{array}$ & Cycling stability & $\begin{array}{l}\text { Capacity } \\
\text { retention } \\
(\%) \\
\end{array}$ & $\begin{array}{c}\text { Capacity } \\
\text { decay rate } \\
(\%) \\
\end{array}$ & Ref. \\
\hline $\mathrm{CP} @ \mathrm{CNF}$ & 200 & $\begin{array}{c}\text { initial capacity } 951.8 \mathrm{mAh} \\
\mathrm{g}^{-1}, 710 \mathrm{mAh} \mathrm{g}^{-1} \text { retained at } \\
0.3 \mathrm{C}\end{array}$ & 74.6 & 0.13 & 1 \\
\hline EUV-CNF & 200 & $\begin{array}{c}\text { initial capacity } 1356 \mathrm{mAh} \\
\mathrm{g}^{-1}, 917 \mathrm{mAh} \mathrm{g}^{-1} \text { retained at } \\
0.2 \mathrm{C}\end{array}$ & 67.6 & 0.16 & 2 \\
\hline $\mathrm{MoO}_{3} @ \mathrm{CNT}$ & 200 & $\begin{array}{c}\text { initial capacity } 1217.7 \mathrm{mAh} \\
\mathrm{g}^{-1}, 755 \mathrm{mAh} \mathrm{g}^{-1} \text { retained at } \\
0.3 \mathrm{C}\end{array}$ & 62 & 0.19 & 3 \\
\hline PANI-GO & 150 & $\begin{array}{c}\text { initial capacity } 1261 \mathrm{mAh} \\
\mathrm{g}^{-1}, 896 \mathrm{mAh} \mathrm{g}^{-1} \text { retained at } \\
0.5 \mathrm{C}\end{array}$ & 73 & 0.18 & 4 \\
\hline $\mathrm{CNTP} / \mathrm{TiO}_{2}$ & 220 & $\begin{array}{l}\text { The capacity of } 791.7 \mathrm{mAh} \\
\mathrm{g}^{-1} \text { after } 30 \text { th cycle, } 575.8 \\
\mathrm{mAh} \mathrm{g}^{-1} \text { retained at } 0.5 \mathrm{C}\end{array}$ & 72.7 & 0.12 & 5 \\
\hline $\mathrm{CoP} / \mathrm{KB}$ & 200 & $\begin{array}{c}\text { initial capacity } 1068.1 \mathrm{mAh} \\
\mathrm{g}^{-1}, 772 \mathrm{mAh} \mathrm{g}^{-1} \text { retained at } \\
0.5 \mathrm{C} \\
\end{array}$ & 72.3 & 0.14 & 6 \\
\hline $\begin{array}{c}\text { MWCNTs/N } \\
\text { CQDs }\end{array}$ & 500 & $\begin{array}{c}\text { initial capacity } 1330.8 \mathrm{mAh} \\
\mathrm{g}^{-1}, 650.7 \mathrm{mAh} \mathrm{g}^{-1} \text { retained } \\
\text { at } 0.5 \mathrm{C}\end{array}$ & 48.9 & 0.1 & 7 \\
\hline $\begin{array}{c}\text { Graphene } \\
\text { oxide/carbon } \\
\text { nanotube } \\
\end{array}$ & 100 & $\begin{array}{c}\text { initial capacity } 1370 \mathrm{mAh} \\
\mathrm{g}^{-1}, 787 \mathrm{mAh} \mathrm{g}^{-1} \text { retained at } \\
0.2 \mathrm{C}\end{array}$ & 58 & 0.42 & 8 \\
\hline $\mathrm{WN} / \mathrm{CC}$ & 500 & $\begin{array}{c}\text { initial capacity } 1337 \mathrm{mAh} \\
\mathrm{g}^{-1}, 814 \mathrm{mAh} \mathrm{g}^{-1} \text { retained at } \\
100 \mathrm{~mA} \mathrm{~g}^{-1}\end{array}$ & 60.9 & 0.078 & $\begin{array}{l}\text { This } \\
\text { study }\end{array}$ \\
\hline
\end{tabular}


Table S2. Impedance parameters, $\mathrm{R}_{\mathrm{e}}, \mathrm{R}_{\mathrm{ct}}$ and $\mathrm{R}_{\mathrm{sf}}$, Warburg factor $(\delta)$ and ion diffusion coefficient (D), obtained from the simulation data in Figure S7a and S7c.

\begin{tabular}{|c|c|c|c|c|}
\hline \multicolumn{2}{|c|}{ Interlayers } & \multirow{2}{*}{$\begin{array}{c}\begin{array}{c}\text { Interlayer-fre } \\
\text { e }\end{array} \\
8.19\end{array}$} & \multirow{2}{*}{$\begin{array}{c}\text { CC interlayer } \\
3.25\end{array}$} & \multirow{2}{*}{$\begin{array}{c}\text { WN/CC interlayer } \\
3.88\end{array}$} \\
\hline \multirow{5}{*}{ Before cycles } & $\mathrm{R}_{\mathrm{e}}(\Omega)$ & & & \\
\hline & $\mathrm{R}_{\mathrm{ct}}(\Omega)$ & 24 & 2.29 & 0.89 \\
\hline & $\mathrm{R}_{\mathrm{sf}}(\Omega)$ & 12 & 6.8 & - \\
\hline & $\begin{array}{c}\delta \\
\left(\Omega \mathrm{cm}^{2} \mathrm{~s}^{-0.5}\right) \\
\end{array}$ & 4.946 & 1.276 & 3.083 \\
\hline & $\mathrm{D}\left(\mathrm{cm}^{2} \mathrm{~s}^{-1}\right)$ & $6.37 \times 10^{-12}$ & $9.57 \times 10^{-11}$ & $1.639 \times 10^{-11}$ \\
\hline \multirow{5}{*}{ After cycles } & $\mathrm{R}_{\mathrm{e}}(\Omega)$ & 7.99 & 4.4 & 4.62 \\
\hline & $\mathrm{R}_{\mathrm{ct}}(\Omega)$ & 56.5 & 2.6 & 1.35 \\
\hline & $\mathrm{R}_{\mathrm{sf}}(\Omega)$ & 81 & 60.9 & 4.57 \\
\hline & $\begin{array}{c}\delta \\
\left(\Omega \mathrm{cm}^{2} \mathrm{~s}^{-0.5}\right) \\
\end{array}$ & 72.612 & 36.435 & 2.9414 \\
\hline & $\mathrm{D}\left(\mathrm{cm}^{2} \mathrm{~s}^{-1}\right)$ & $2.95 \times 10^{-14}$ & $1.17 \times 10^{-13}$ & $1.8 \times 10^{-11}$ \\
\hline
\end{tabular}




\section{References}

1. Li, Q.; Liu, M.; Qin, X.; Wu, J.; Han, W.; Liang, G.; Zho, D.; He, Y. B.; Li, B.; Kang, F., Cyclized-polyacrylonitrile Modified Carbon Nanofiber Interlayer Enabling Strong Trapping For Polysulfides in Lithium-sulfur Battery. J. Mater. Chem. A 2016, 4, 12973-12980.

2. Wu, K.; Hu, Y.; Shen, Z.; Chen, R.; He, X.; Cheng, Z.; Pan, P., Highly Efficient and Green Fabrication of a Modified C Nanofiber Interlayer for High-performance Li-S Batteries. J. Mater. Chem. A 2018, 6, 2693-2699.

3. Luo, L.; Qin, X.; Wu, J.; Liang, G.; Li Q.; Liu, M.; Kang, F.; Chen, G.; Li, B., Interwoven $\mathrm{MoO}_{3} @ \mathrm{CNT}$ Scaffold Interlayer for High-performance Lithium-sulfur Batteries. J. Mater. Chem. A 2018, 6, 8612-8619.

4. Yin, L.; Dou, H.; Wang, A.; Xu, G.; Nie, P.; Chang, Z.; Zhang, X., A Functional Interlayer as a Polysulfides Blocking Layer for High-performance Lithium-sulfur Batteries. New J. Chem. 2018, 42, 1431-1436.

5. Xu, G.; Yuan, J.; Tao, X.; Ding, B.; Dou, H.; Yan, X.; Xiao, Y.; Zhang, X., Absorption Mmechanism of Carbon-nanotube Paper-titanium Dioxide as a Multifunctional Barrier Material for Lithium-sulfur Batteries. Nano Res. 2015, 8, 3066-3074.

6. Chen, X.; Ding, X.; Wang, C.; Feng, Z.; Xu, L.; Gao, X.; Zhai, Y.; Wang, D., Multi-shelled CoP Nanospheres Modified Separator for Highly Efficient Li-S Batteries. Nanoscale 2018, 10, 13694-13701.

7. Pang, Y.; Wei, J.; Wang, Y.; Xia, Y., Synergetic Protective Effect of the Ultralight MWCNTs/NCQDs Modified Separator for Highly Stable Lithium-sulfur Batteries. Adv. Energy Mater. 2018, 8, 1702288-1702299.

8. Huang, J. Q.; Xu, Z. L.; Abouali, S.; Garakani, M. A.; Kim, J. K., Porous Graphene Oxide/carbon Nanotube Hybrid Films as Interlayer for Lithium-sulfur Batteries. Carbon 2016, 99, 624-632. 